\title{
Correction: $\mathrm{Se}_{2} \mathrm{Mo}_{10} \mathrm{~V}_{3}$, a heteropoly compound containing selenium, inhibits tumor growth
}

\author{
Hong-Ning Zhang ${ }^{1}$, Wei-Li Feng ${ }^{2}$, Chun-Na An ${ }^{3}$ and Wen-Guang Li ${ }^{4}$ \\ ${ }^{1}$ Department of Pharmacology, School of Basic Medical Sciences, Capital Medical University, Beijing 100069, China \\ ${ }^{2}$ Department of Pharmacology, School of Basic Medical Sciences, Qinghai University, Xining 810001, China \\ ${ }^{3}$ Department of Pharmacology, School of Basic Medical Sciences, North China University of Science and Technology, Tangshan \\ 063009, China \\ ${ }^{4}$ Department of Pharmacology, School of Basic Medical Sciences, Lanzhou University, Lanzhou 730000, China \\ Published: November 23, 2018
}

Copyright: Zhang et al. This is an open-access article distributed under the terms of the Creative Commons Attribution License 3.0 (CC BY 3.0), which permits unrestricted use, distribution, and reproduction in any medium, provided the original author and source are credited.

This article has been corrected: The correct information of the Author contributions and Funding is given below:

\section{Author contributions}

H.-N. Z. performed the experiments. W.-L. F. helped to perform the in vitro experiment. C.-N. A. wrote the manuscript, prepared the figures and supplied publication costs. W.-G. L. designed the project. All authors reviewed the manuscript.

\section{FUNDING}

This work was supported by Science and Technology Program of Beijing Municipal Education Commission (KM201810025002) and Health Science Key Program of Hebei Province (ZD20140029).

Original article: Oncotarget. 2017; 8:67871-67877. https://doi.org/10.18632/oncotarget.18909 